\title{
Microscopic Image Segmentation of Chinese Herbal Medicine Based on Region Growing Algorithm
}

\author{
Qing Liu, Lijun Zhang \\ School of Physics \& Information Science \\ Tianshui Normal University \\ Tianshui, Gansu, 741001, China \\ xdlq@163.com; 47216162@qq.com
}

\author{
Xiping Liu \\ Department of Basic Medical Sciences \\ Gansu University of TCM \\ Lanzhou, Gansu, 730000, China \\ lxpd-257@163.com
}

\begin{abstract}
In order to effectively separate the target region of the microscopic image of Chinese Herbal Medicine (CHM), and lay the foundation for the subsequent image recognition processing, a microscopic image segmentation method of CHM by using region growing (RG) algorithm is put forward based on the characteristics of the plant microscopic images. Firstly, the CHM microscopic images with different cell structure are regarded as a multi-dimensional matrix to process and established seed label matrix. Secondly, in a given region threshold conditions, the different seed growth points are selected to segmented the different images. Finally, given a fixed growth points, the microscopic images are processed by choosing a different threshold. The experimental results show that CHM image segmentation threshold and seed selection decide the image target extraction. For different CHM images, according to a certain method, the better image segmentation results can be achieved in the case to obtain a suitable threshold value using image information and the seed point adjustment.
\end{abstract}

Keywords- Image segmentation; Region growing; Microscopic image; Chinese herbal medicine

\section{INTRODUCTION}

The quality of the original Chinese Herbal Medicine (CHM) impacts directly on the treatment of disease. The selection of the raw material of CHM attaches great importance to the field of Chinese medicine and is one of the research topics. Because the degradation, fake shoddy and other factors of CHM affect its quality and clinical curative effect, the identification, distinction and control to Chinese herbal medicine is becoming increasingly important. The traditional CHM distinction mainly base on subjective experience. Chemical and physical CHM identification must rely on some instruments [1]. The detection and identification of CHM based on the information technology is the new development direction [2]. The modern testing and identification of CHM by utilizing modern image processing is one of the modern processing technology development prospects in the IT. The target region of the microscopic images must be effectively extracted using image processing technology for the recognition and identification of CHM microscopic images. Then the effective image segmentation technique will be taken to the microscopic images of CHM. Image segmentation of CHM has a very important role in pharmacology for traditional Chinese medicine. It can be provided the visible information to the analysis of the physical and chemical properties in the cellular structure level by using the image segmentation to the traditional Chinese medicine on tissue microstructure image object extraction. In addition, the identification and identification of CHM quality with its morphological features will be achieved through the effective target region segmentation of the CHM microscopic image. The image segmentation technique is applied to the modern Chinese herbal medicine identification, which can overcome the limitations of the traditional experience and can extract the useful information in the image target. This method can use the new mathematical tools and the information processing technology to the identification of CHM and has the advantages of simple operation, measurement accuracy and repeatability [3].

With the development of image processing technology, it can convert the original images into more abstract and more compact form that the feature extraction and the target expression based on image segmentation, which makes it possible that more in-depth image analysis and understanding [4]. Image segmentation is the most important issue in image analysis and pattern recognition, and is one of the difficult problems of image processing [5]. Image segmentation is a very challenging task in computer vision and image understanding. There are four kind of different methods to image segmentation, that is threshold, edge, region and hybrid method. But there is not a good standard method to any image segmentation [6].

Because CHM involves wide range, the different CHM microscopic images show the different regional cross tissue distribution. Even for the same kind of CHM, there is the different regional shape feature due to its growing area and distribution is different. So the regional method is a more commonly used segmentation method to the microscopic images segmentation of CHM. The tissue target in microscopic image is segmented and the given characteristic value is extracted by using image segmentation technology for CHM. CHM image recognition accuracy can be enhanced. Region growing method is one of the important region segmentation methods. But the dependence of the seed selection is strong, and the growth threshold selection 
is very sensitive to the noise interference [7]. According to the characteristics of microscopic images of CHM, the images are segmented in the choice of different growth thresholds and different seed points. It is effectively solved that the strong dependence of the regional growth seeds point and the different segmentation threshold (oversegmentation and under-segmentation), then better effect of segmentation is obtained.

\section{REGION GROWING ALGORITHM AND ITS IMPLEMENTATION TO MICROSCOPIC IMAGE OF CHM}

Region growing (RG) is a kind of typical serial segmentation technique, and is a very important image segmentation method in artificial intelligent field of computer vision research. RG is to have similar properties of the set of pixels form a region. The basic idea of RG algorithm is that a seed pixel point as the starting point is found in each segmented region, and then the regional growth is carried on according to the similarity criterion until no merging point [8]. So back and forth, until no more pixels can be combined, such a region is formed. The image space relation based on region segmentation method, not only take into account the similarity of the pixel, but also considering the spatial region adjacency.

The key of this method lies in how to select the appropriate seed points, to develop the growth criteria and to determine the termination condition according to the different images, and then the purpose of the adaptive image segmentation can be achieved. In general, regional growth will stop in the absence of pixel or no regional added growth regional conditions.

\section{A. Selection of the Seed Point}

The seed is the starting point of the regional growth. The appropriate seed selection is successfully relation to the whole growth processing, and also relate to the effect of the final image segmentation. The seed points may be a single pixel with a certain gray value or a small area combined with many pixels, its selection is based primarily on the characteristics of the image and to achieve the purpose. In this paper, the gray image information is regarded as the basis selected seed point. However, because the different characteristics of the different CHM, its microscopic images have different gray value and distribution, so the different gray values are selected according to different image. Provided that the microscopic image is $f(x, y)$, in order to improve seed point representation, the initial seed points near the residential area of the $\mathrm{N}$ points are selected, and the average gray value of the $\mathrm{N}$ pixel points are calculated as the final gray value of the seed point. It can be expressed with $S$.

$$
S=\frac{1}{N} \sum_{k}^{N} f_{k}(x, y)
$$

\section{B. Region growing Standars}

The determination of the growth standards is a central validity issue related to the algorithm in region growing processing [9]. The pixels based on the regional homogeneity can be incorporated into the same region, which must meet certain criteria.

In the multi-dimensional space, the Euclidean distance of any two positions can be defined as equation (2)

$$
D_{E}^{2}=\sum_{d=1}^{n}\left(L_{d}(x, y)-L_{d}\left(x^{\prime}, y^{\prime}\right)\right)^{2}
$$

In the above formula, $D_{E}$ is the Euclidean distance between the any two points; $d$ is the space dimension; $L_{d}(x, y)$,

$L_{d}\left(x^{\prime}, y^{\prime}\right)$ is the arbitrary two Points of $d$ dimensional space.

Euclidean distance based on seed point in RGB space can be written as

$$
\begin{aligned}
& D_{E}^{2}\left(L(x, y), L_{s}\left(x^{\prime}, y^{\prime}\right)\right)= \\
& \quad\left(R_{L}-R_{L s}\right)^{2}+\left(G_{L}-G_{L s}\right)^{2}+\left(B_{L}-B_{L s}\right)^{2}
\end{aligned}
$$

In the formula (3), $L(x, y), L_{s}\left(x^{\prime}, y^{\prime}\right)$ is non-seed points and seed points; $R_{L}, G_{L}, B_{L}$ is the brightness values of non-seed points in RGB, and $R_{L s}, G_{L s}, B_{L s}$ is the brightness values of seed points in RGB, respectively.

If $L_{s}$ is the seed point of the region $W$, a threshold value $T$ is given, then

$$
\left\|D_{E}\left(L(x, y), L_{s}\left(x^{\prime}, y^{\prime}\right)\right)\right\|\left\{\begin{array}{l}
\leq T, L \in W \\
>T, L \notin W
\end{array}\right.
$$

Equation (4) is the judging standards of the regional growth and the end.

\section{Region growing method}

There are two kinds of regional growth pattern, that is 4-connected way and 8-connected way, it can be expressed with the coordinate in a two-dimensional plane. Regional connectivity is an important condition of region growing, which affects the core structure of the segmentation algorithm. Therefore, the connectivity conditions need to be strictly ensured in region growing process.

Taking into account the relevance of the pixel coordinates, equation (4) can be made the following improvements

$$
\left\|D_{E}\left(L\left(x^{\prime}+u, y^{\prime}+v\right), L_{s}\left(x^{\prime}, y^{\prime}\right)\right)\right\|\left\{\begin{array}{l}
\leq T, L \in W \\
>T, L \notin W
\end{array}\right.
$$

In the above formula, $u=-1,0,1 ; v=-1,0,1$.

Whether the four communication mode or eight communication mode is adopted, the neighboring pixels around the seed points can be detected and the regional continuity and the integrity is ensured by taking $u, v$ value as conditions. In the growth process, the local loop expansion method is used, so that the segmentation efficiency is greatly improved, and the segmentation algorithm has been further optimized.

Region growing segmentation algorithm concrete steps are as follows 
Step 1: the image is sequentially scanned to find a seed point and located the pixel position $\left(x_{0}, y_{0}\right)$ of the point.

Step 2: 8 neighborhood pixels point $(x, y)$ is considered when $\left(x_{0}, y_{0}\right)$ is seen as the center point, and the average grayscale value of these pixels is calculated. The point $(x, y)$ meet growth criteria will be merged with $\left(x_{0}, y_{0}\right)$, at the same time, the point $(x, y)$ will be pushed onto the stack.

Step 3: another pixel is taken out from the stack and regarded as $\left(x_{0}, y_{0}\right)$, and then continue with step 2 .

Step 4: When the stack is empty, return to step 1.

Step 5: Repeat steps 1-4 until each pixel of the image is belonged to a given region, then stop the growth process.

\section{EXPERIMENTAL RESULTS AND ANALYSIS}

In this experiment, the partial authentic plants of Chinese herbal medicines produced in Gansu province, Such as Radix sileris, Rhizoma dryopteris, Radices rehmanniae,etc, were selected as the original materials. Those microscopic images taken under an electron microscope were obtained as the experimental objects based on early processing to CHM. The single target microscopic images and the multi-target microscopic images of CHM were segmented using the proposed region growing algorithm, and experimental study of different seed points selection and different threshold settings for these images were carried on.

(1) Segmented experiment of the single target image of Radix sileris was used RG. The image size of Radix sileris is $80 \times 80$. The experimental results are showed in the figure 1 and figure 2 .

(2) The multi-target microscopic images of Rhizoma dryopteris of CHM was segmented utilizing RG. The image size of Rhizoma dryopteris is $256 \times 256$.The segmentation results are showed in figure 3 and figure 4 .

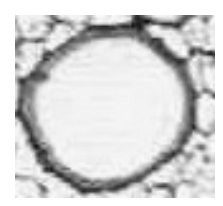

(a) Original image

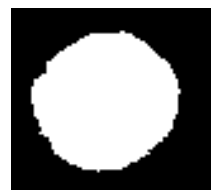

(d) $T=40$

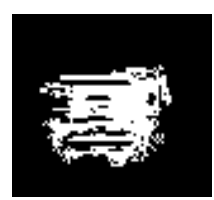

(b) $T=2$

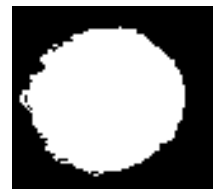

(e) $T=100$

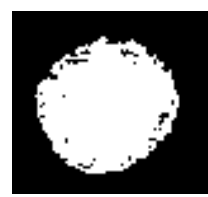

(c) $T=10$

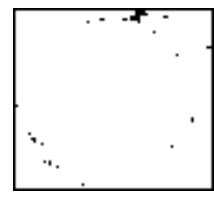

(f) $T=200$
Figure 1. Segmentation results of the single target image of Radix sileris set the different threshold values $T$ at fixed growing point $\left(x_{0}, y_{0}\right)=(38,36)$

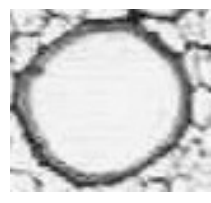

(a) Original image

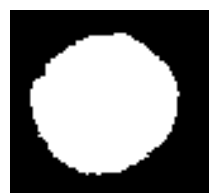

(d) $\left(x_{0}, y_{0}\right)=(61,33)$

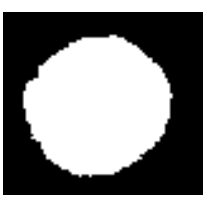

(b) $\left(x_{0}, y_{0}\right)=(37,37)$

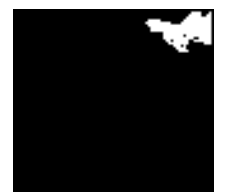

(e) $\left(x_{0}, y_{0}\right)=(7,74)$

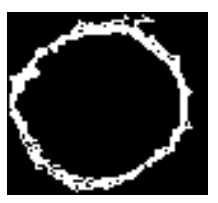

(c) $\left(x_{0}, y_{0}\right)=(8,49)$

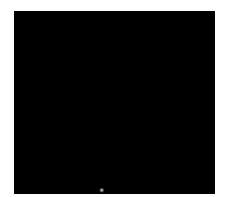

(f) $\left(x_{0}, y_{0}\right)=(71,35)$
Figure 2. Segmentation results of the single target image of Radix sileris selected the different growing point $\left(x_{0}, y_{0}\right)$ at the fixed threshold values $T=40$

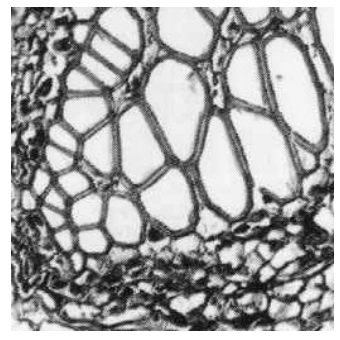

(a) Original image

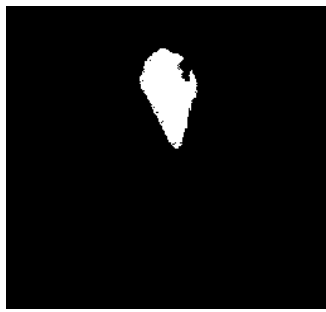

(c) $T=20$

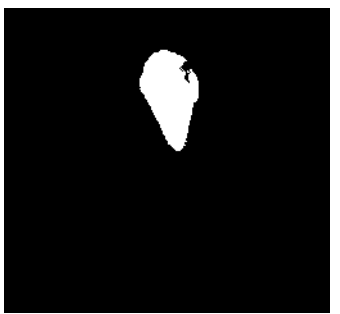

(e) $T=60$

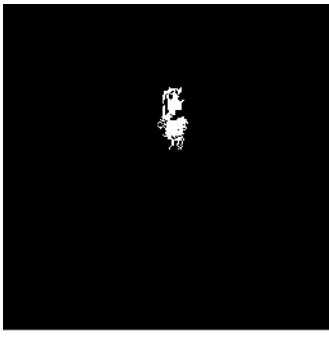

(b) $T=2$

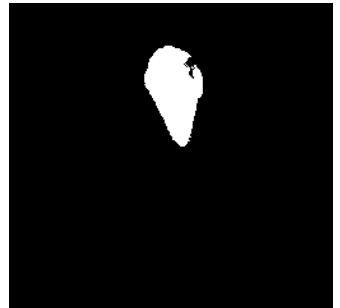

(d) $T=40$

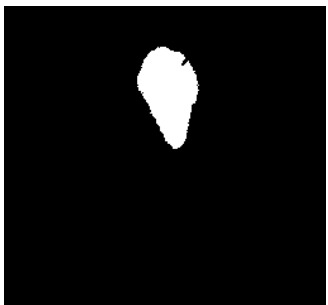

(f) $T=100$
Figure 3. Segmentation results of the multi-target image of Rhizoma dryopteris set the different threshold values $T$ at fixed growing point $\left(x_{0}, y_{0}\right)=(76,132)$ 


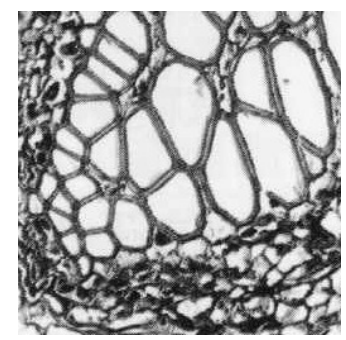

(a) Original image

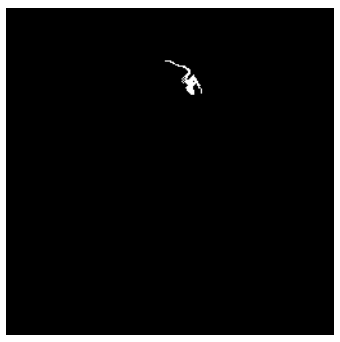

(c) $\left(x_{0}, y_{0}\right)=(57,145)$

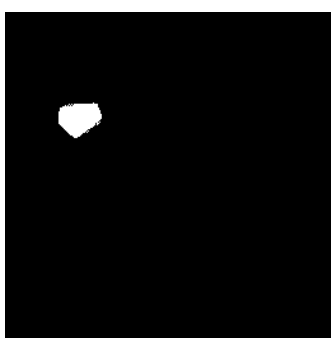

(e) $\left(x_{0}, y_{0}\right)=(87,65)$

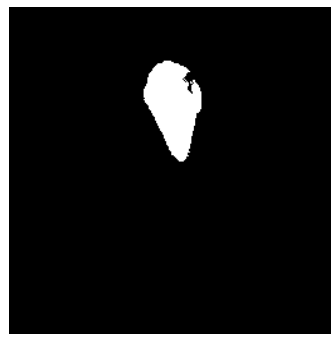

(b) $\left(x_{0}, y_{0}\right)=(80,136)$

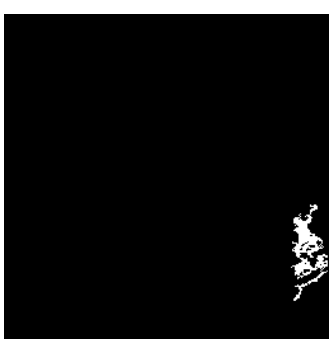

(d) $\left(x_{0}, y_{0}\right)=(179,239)$

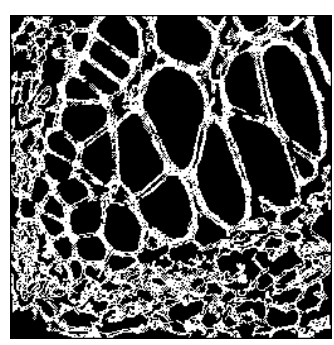

(f) $\left(x_{0}, y_{0}\right)=(126,142)$
Figure 4. Segmentation results of the multi-target image of Rhizoma dryopteris selected the different growing point $\left(x_{0}, y_{0}\right)$ at the fixed threshold values $T=40$

From the experiment can be seen that the different threshold settings will cause the different segmentation results in the choice of the appropriate seed point to the image segmentation of the single target image or the multiple target in Figure 1 and in figure 3. Specifically, the white area gradually increases with the increase of the threshold of the single target segmentation from (b) to (f) in figure 1, that is, the target area to be extracted is gradually increased. Each figure is compared with the original can be found that the figure (d) is closest to the original image (a), that is to say figure (d) obtained the best segmentation. The results of segmentation object extraction degree determined by the threshold selection can also be obtained from the multiple target microscopic image segmentation of CHM in figure 3 (b)-(f). So too much threshold makes image oversegmentation and too small threshold can not effectively control the segmentation environment.

Figure 2 and figure 4 reflects the segmentation result influence of the different seed point's selection for the single target microscopic image of Radix sileris and the multi target microscopic image of Rhizoma dryopteris under a given threshold. More representative of the seed point is selected for the image segmentation of the single target region in figure 2. The experimental results indicate that the regular segmentation graph can be got when the relatively central location in image cell structure is selected, and formed a similar to the cell wall map image when the cell wall structure position is chosen. The different segmented image can be formed through continuously adjusting the seed point at the threshold $T=40$ in figure 4 . The multiple target area can be extracted and the ideal segmentation effect can be achieved when the seed point is set as $\left(x_{0}, y_{0}\right)=(126,142)$. The segmentation results are shown in figure 4 (f).

\section{CONCLUSIONS}

In order to realize the scientific, rapid and effective traditional Chinese medicine detection, microscopic image database of CHM must be established and the systematic classification and identification will be achieved with the help of modern information processing technology. In this paper, a region growing algorithm of image target extraction is presented according to the characteristics of the microscopic images of CHM. The image segmentation is carried out to the part of single target image and multi-target image in the given different threshold and different seed points by using the RG technology. This algorithm has a favorable capability to carve up characteristics and maintain the edges, texture and details of images, has higher precision in image segmentation and can be more adaptability. This method is applied to establish the image database of CHM, which can greatly improve the accuracy of the data in the database. The establishment of image retrieval system will be helpful for herbal medicinal species identification and authenticity identification.

\section{ACKNOWLEDGMENT}

This work is supported by National Natural Science Foundation of P. R. China (81260525), Natural Science Foundation of Gansu Province (1010RJZE028) and “QingLan” Talent Engineering Funds by Tianshui Normal University

\section{REFERENCES}

[1] Qing Liu, Xiping Liu, Shuangwang Han,et al. "Detection and identification of Chinese herbal medicines based on modern processing technology," Health Vocational Education, Vol.30. pp. 154-155, 2012.

[2] W. Lu, X.Y. Qin, W. Chen, S.T. "Chen.:Automatic Hotspots Recognition and Trends Prediction in Traditional Medicine”. Proc. IEEE Symp. IT in Medicine and Education (ITME), IEEE, Press, pp. 753-756, 2008.

[3] Wanxiang Zhang, Qichan Pang, Jing Zhao,et al. "Self-adaptive region growing algorithm to segment images of spectral imaging for TCM assessment,” Journal of Applied Optics, Vol.31. pp. 78-82, 2010.

[4] D.P Ming, J.H Luo, C.H Zhou. "Researeh on high resolution remote sensing image segmentation methods based on features and evaiuation of algorithms," GEOInformation Science, Vol.18. pp. 103109, 2006.

[5] Qing Liu, Luping $\mathrm{Xu}$, Yide $\mathrm{Ma}$, et al. "Automated image segmentation using the ULPCNN model with ultra-fuzzy entropy," Journal of XIDIAN University, Vol.37. pp. 817-824, 2010. 
[6] Frank Y S, Shouxian Cheng. "Automatic seeded region growing for color image segmentation,” Image and Vision Computing, Vol.23, pp. 877-886,2005.

[7] Rajasvaran Logeswaran, Chikkannan Es Waran. "Stone detection in MRCP images using con- trolled region growing," Computers in Biology and Medicine,Vol.37, pp.1084-1091,2007.

[8] Zhengguo Li, Chungan Li, Hua-bing Dai. "Segmentation method for forest zone remote sensing imag based-RG algorithm," Guangxi Forestry Scienc, Vol.37, pp.66-70,2008.

[9] S.Y.Wang . "Symmetric region growing," IEEE Transactions on Image Processing, Vol.12, pp.1007-1015,2003. 